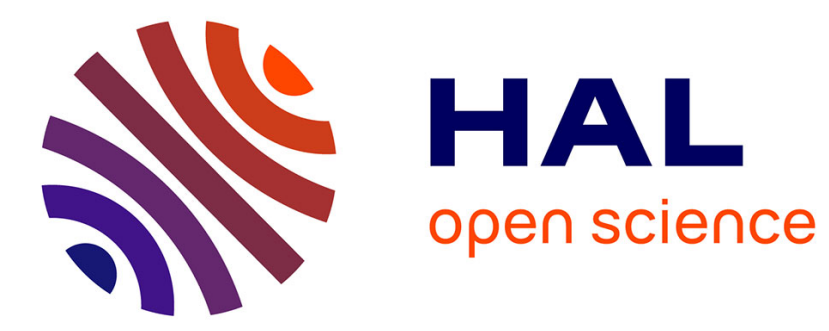

\title{
Colossal magneto-resistive relaxation effects in La0.9Ce0.1Fe12B6
}

\author{
L. V. B. Diop, O. Isnard
}

\section{To cite this version:}

L. V. B. Diop, O. Isnard. Colossal magneto-resistive relaxation effects in La0.9Ce0.1Fe12B6. Journal of Applied Physics, 2021, 129 (24), pp.243902. 10.1063/5.0055885 . hal-03325760

\section{HAL Id: hal-03325760 \\ https://hal.science/hal-03325760}

Submitted on 25 Aug 2021

HAL is a multi-disciplinary open access archive for the deposit and dissemination of scientific research documents, whether they are published or not. The documents may come from teaching and research institutions in France or abroad, or from public or private research centers.
L'archive ouverte pluridisciplinaire HAL, est destinée au dépôt et à la diffusion de documents scientifiques de niveau recherche, publiés ou non, émanant des établissements d'enseignement et de recherche français ou étrangers, des laboratoires publics ou privés. 


\title{
Colossal magneto-resistive relaxation effects in $\mathrm{La}_{0.9} \mathrm{Ce}_{0.1} \mathrm{Fe}_{12} \mathrm{~B}_{6}$
}

\author{
L.V.B. Diop ${ }^{1 \dagger}$ and O. Isnard ${ }^{2}$ \\ ${ }^{1}$ Université de Lorraine, CNRS, IJL, F-54000 Nancy, France \\ ${ }^{2}$ Université Grenoble Alpes, CNRS, Institut NEEL, 25 rue des martyrs, BP166X, F-38042 Grenoble,
}

France

The study of the magnetic, electronic transport and magnetotransport properties of $\mathrm{La} 0.9 \mathrm{Ce}_{0.1} \mathrm{Fe}_{12} \mathrm{~B}_{6}$ itinerant-electron system has been performed by combining magnetization, electrical resistivity and magnetoresistance experiments. Along with the antiferromagnetic (AFM) ordering at $T_{\mathrm{N}}=35 \mathrm{~K}$, two consecutive magnetic transformations antiferromagneticferromagnetic (AFM-FM) and ferromagnetic-paramagnetic (FM-PM) occur upon heating under certain magnetic field values. At fixed temperatures, it is revealed that both AFM and PM phases can be converted into the FM phase irreversibly and reversibly via a first-order metamagnetic transition associated with a large hysteresis. Below $8 \mathrm{~K}$, the metamagnetic transition is discontinuous manifesting itself by multiple sudden jumps in magnetoresistance and magnetization. A giant negative magnetoresistance effect of about $-78 \%$ is found. We further demonstrate that the time dependencies of the electrical resistivity and the magnetization exhibit colossal spontaneous steps after an incubation time in conditions where both the applied magnetic field and temperature are constant. Another intriguing observation in the phase diagram is the presence of a critical point at the crossover of the three distinct PM, FM, and AFM magnetic states.

\section{INTRODUCTION}

The itinerant-electron metamagnetic system $\mathrm{LaFe}_{12} \mathrm{~B}_{6}$ occupies a special place among rare-earth iron-rich intermetallic compounds; it exhibits uncommon magnetic behavior and many intriguing physical properties. Interestingly, discontinuous and unconventional staircaselike metamagnetic phase transitions were recently discovered in $\mathrm{LaFe}_{12} \mathrm{~B}_{6}$ compound $^{1-4}$. This peculiar multistep behavior is featured by extremely sharp magnetization steps, followed by plateaus leading to an unusual and unique avalanche-like magnetization process. According to neutron powder diffraction investigation, $\mathrm{LaFe}_{12} \mathrm{~B}_{6}$ displays an amplitude-modulated antiferromagnetic spin configuration described by a magnetic propagation vector $\mathbf{k}=(1 / 4,1 / 4$, $1 / 4)$, especially weak Fe moment $\left(0.43 \mu_{\mathrm{B}}\right)$ in the magnetically ordered ground state. Moreover

\footnotetext{
† leopold.diop@univ-lorraine.fr
} 
remarkably low Néel temperature $T_{\mathrm{N}}=36 \mathrm{~K}$ for an Fe-rich intermetallic phase is observed together with unconventional magnetic behaviors including a critical point in the complex magnetic phase diagram ${ }^{1}$, both inverse and normal magnetocaloric phenomenon ${ }^{5}$, giant spontaneous magnetization steps ${ }^{3}$, and large pressure effects ${ }^{6}$. These singular features not only offered the development of experiments under extreme conditions and theoretical models for a deeper insight into the fascinating physics underlying the striking behavior of this compound ${ }^{6-}$ ${ }^{10}$, but also highlighted the potential interest of $\mathrm{LaFe}_{12} \mathrm{~B}_{6}$ system in future low-temperature energy technologies. The ternary compound $\mathrm{LaFe}_{12} \mathrm{~B}_{6}$ constitutes an exceptional playground for materials physics owing to the extreme sensitivity of its physical properties to reasonably weak external pressure ${ }^{6}$ and chemical substitution ${ }^{4}$.

Among the $R T_{12} \mathrm{~B}_{6}$ family (where $R$ is a rare-earth atom and $T$ stands for a $3 d$ transition metal element $\mathrm{Co}$ or $\mathrm{Fe}$ ), $\mathrm{LaFe}_{12} \mathrm{~B}_{6}$ is the sole stable Fe-based compound of the 1:12:6 ternary system $^{9-10}$. On the other hand, the $R \mathrm{Co}_{12} \mathrm{~B}_{6}$ intermetallics are stable along the entire rare-earth series (except for Europium) ${ }^{11}$. Although being the first Fe-based member of the $R T_{12} \mathrm{~B}_{6}$ family to be identified, $\mathrm{NdFe}_{12} \mathrm{~B}_{6}$ alloy is metastable ${ }^{12}$. At room temperature, the $R T_{12} \mathrm{~B}_{6}$ series of compounds crystallizes in the rhombohedral $\mathrm{SrNi}_{12} \mathrm{~B}_{6}$-type structure with $R \overline{3} m$ crystallographic space group ${ }^{13-15}$. In the crystal structure, there are two crystallographically inequivalent positions for $T$ atoms (18g and 18h). The $R$ and B atoms occupy the $3 a$ and $18 h$ Wyckoff sites, respectively. $\mathrm{LaFe}_{12} \mathrm{~B}_{6}$ compound is unique among the ternary system $R T_{12} \mathrm{~B}_{6}$ exhibiting an antiferromagnetic ground state with an ordering temperature much smaller than the Curie point of the Co-based $R \mathrm{Co}_{12} \mathrm{~B}_{6}$ ferro- $(R=\mathrm{Y}$, La-Sm) or ferri- $(R=\mathrm{Gd}-\mathrm{Tm})$ magnets $\left(T_{\mathrm{C}}=134-162 \mathrm{~K}\right)^{11}$. The Néel temperature of $\mathrm{LaFe}_{12} \mathrm{~B}_{6}$ is an order of magnitude smaller when compared to the magnetic ordering temperature of any rare-earth iron-rich binary alloy. Extraordinary magnetotransport properties have been most recently discovered in $R \mathrm{Co}_{12} \mathrm{~B}_{6}$ intermetallics with $R=\mathrm{Y}, \mathrm{Gd}$ and $\mathrm{Ho}^{16}$.

In the present paper, we carry out a thorough experimental investigation of the La $0.9 \mathrm{Ce}_{0.1} \mathrm{Fe}_{12} \mathrm{~B}_{6}$ model system by a combination of magnetization, electrical resistivity and magnetoresistance measurements. The paper is organized as follows : after a brief description of the experimental methods (Section II), we present the results of our studies of the magnetic properties (Section III.A) and transport properties (Section III.B). This is followed by a conclusion (Section IV).

\section{METHODS}

The synthesis and structural characterization of the $\mathrm{La} 0.9 \mathrm{Ce}_{0.1} \mathrm{Fe}_{12} \mathrm{~B}_{6}$ polycrystalline sample are described in Ref. 4. Magnetization experiments were carried out on a powder sample 
using an extraction-type magnetometer. Temperature, magnetic field and time dependencies of the magnetization were measured in applied fields of up to $10.5 \mathrm{~T}$. A detailed description of the magnetometer can be found in Ref. 17. Magnetic data were corrected for the presence of the minor ferromagnetic $\mathrm{Fe}_{2} \mathrm{~B}$ secondary phase. The sample for the electrical resistivity and magnetoresistance measurements was cut in parallelepiped form using diamond saw and subsequently smooth and flat surfaces were prepared by polishing. The electrical connections on the sample surface were made by fixing thin platinum wires using silver paste. The measurements were undertaken using the conventional four-point contact method at a constant dc current of $10 \mathrm{~mA}$ at temperatures ranging between 2.5 and $125 \mathrm{~K}$ in a superconducting magnet producing a maximum magnetic field of $8 \mathrm{~T}$. The magnetic field was applied perpendicular to the current orientation $(H \perp i)$. In order to get rid of possible thermals, the dc electrical current was applied in opposite polarities at each measurement point.

\section{RESULTS AND DISCUSSION}

\section{A. Magnetic properties}

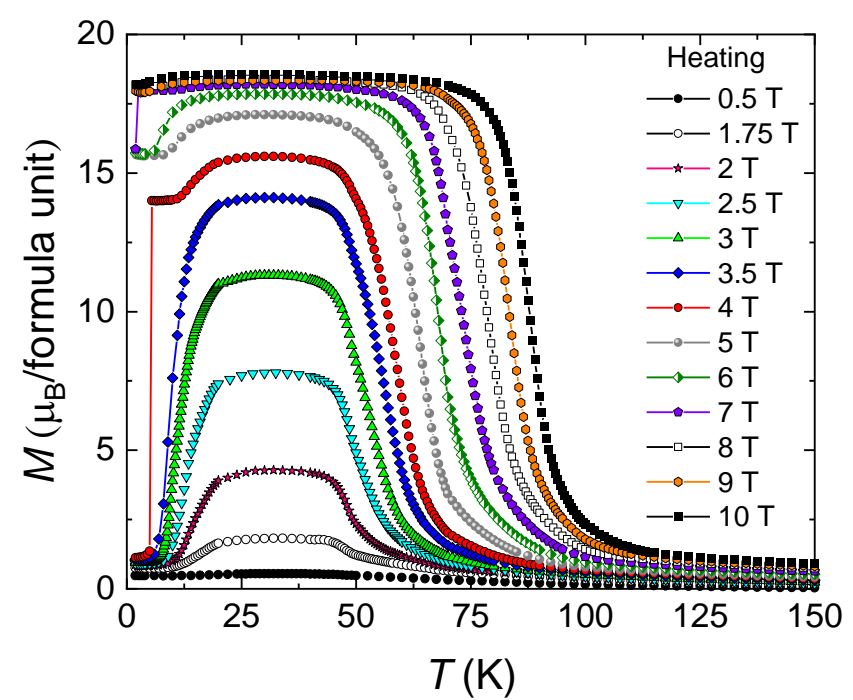

FIG. 1. Temperature dependence of the magnetization of $\mathrm{ZFC} \mathrm{La}{ }_{0.9} \mathrm{Ce}_{0.1} \mathrm{Fe}_{12} \mathrm{~B}_{6}$ recorded upon heating in various magnetic fields.

The thermomagnetic data, $M(T)$, of the zero-magnetic field cooled (ZFC) $\mathrm{La} 0.9 \mathrm{Ce}_{0.1} \mathrm{Fe}_{12} \mathrm{~B}_{6}$ collected upon warming under various applied fields, are plotted in Fig. 1 . The low-field temperature-dependent magnetization curve $(\mu \mathrm{H}=0.5 \mathrm{~T})$ shows a small peak around the Néel temperature $T_{\mathrm{N}}=35 \mathrm{~K}$ indicating a transition from an antiferromagnetic (AFM) to a paramagnetic (PM) state. When the applied field exceeds $7 \mathrm{~T}, \mathrm{La} 0 .{ }_{9} \mathrm{Ce}_{0.1} \mathrm{Fe}_{12} \mathrm{~B}_{6}$ is in the fully ferromagnetic (FM) polarized phase, and therefore the thermomagnetic curves reflect the magnetic phase transformation from FM to PM state. The isofield magnetization curves taken 
in magnetic fields of $\mu_{0} H=5,6$ and $7 \mathrm{~T}$ indicates that a large proportion, $\sim 85 \%$, of the La0.9 $\mathrm{Ce}_{0.1} \mathrm{Fe}_{12} \mathrm{~B}_{6}$ compound is converted into a $\mathrm{FM}$ phase at $2 \mathrm{~K}$, and $\sim 15 \%$ remains in the initial AFM ground state.

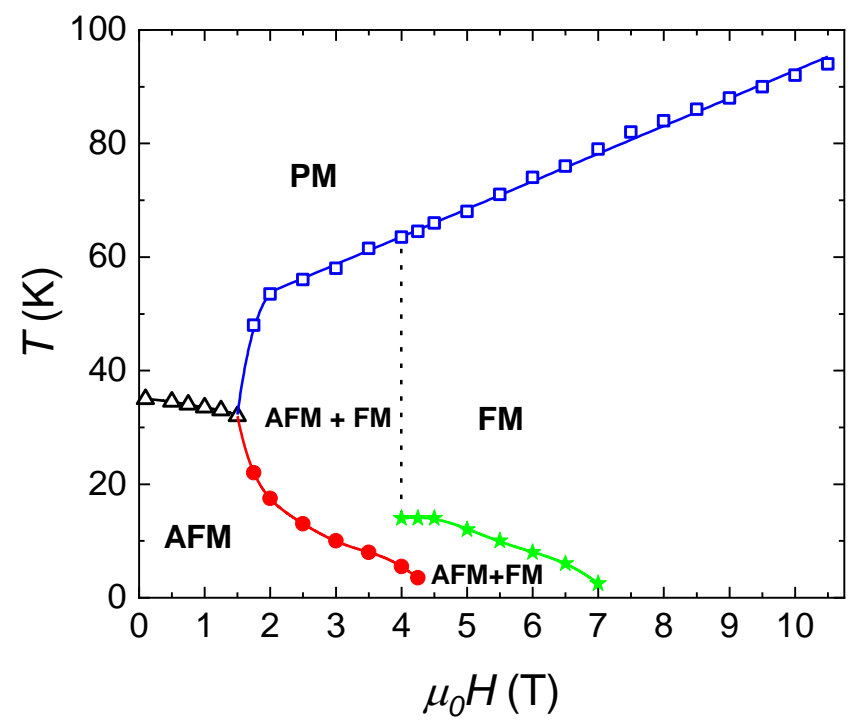

FIG. 2. Magnetic phase diagram of $\mathrm{La}_{0.9} \mathrm{Ce}_{0.1} \mathrm{Fe}_{12} \mathrm{~B}_{6}$, as derived from ZFC thermomagnetic measurements.

Yet, the magnetization displays considerably different thermal behavior when performed in applied field ranging from 1.75 to $3.5 \mathrm{~T}$. These $M(T)$ measurements manifest a bell-shaped anomaly and two magnetic events occur sequentially during warming. The first one refers to an AFM-FM transformation at low temperatures, and the second one corresponds to FM to PM phase transition at high temperatures. As can be seen from Fig.1, a plateau forms around the center of the bell-shaped anomaly $(\approx 32 \mathrm{~K})$. Note that both the width of the anomaly and the height of the plateau grow with increasing magnetic field, i.e., the amount of the induced-FM component is strongly dependent on the strength of applied field, which favors the FM order over the AFM phase. $\mathrm{La}_{0.9} \mathrm{Ce}_{0.1} \mathrm{Fe}_{12} \mathrm{~B}_{6}$ gets transformed only partially into the FM phase in this magnetic field interval. Our interpretation of these results is that throughout this magnetic transformation, both magnetically AFM and FM ordered states coexist, hence creating a magnetically inhomogeneous state (magnetic-phase-segregated state), a typical behavior for first-order magnetic phase transition. In a $4 \mathrm{~T}$ magnetic field, the thermomagnetic curve exhibits an abrupt jump where the magnetization changes drastically from 1.37 to $14.05 \mu_{\mathrm{B}} /$ formula unit. This spectacular rise in magnetization by $925 \%$, when temperature is increased by $0.5 \mathrm{~K}$, is associated with the instantaneous development of the FM phase. A similar bell-shaped anomaly was evidenced in the parent compound $\mathrm{LaFe}_{12} \mathrm{~B}_{6}$ within the magnetic field range between 4.75 and $7 \mathrm{~T}^{1,2}$. 
The magnetic phase diagram of $\mathrm{La} 0 .{ }_{9} \mathrm{Ce}_{0.1} \mathrm{Fe}_{12} \mathrm{~B}_{6}$ is depicted in Fig. 2. The magnetic ordering temperatures were derived from the thermomagnetic curves of Fig. 1. The Curie temperature (FM-PM), $T_{\mathrm{C}}$, was defined as the abscissa of the crossing point of the hightemperature part of the extended baseline and the linear portion of the ZFC $M(T)$ curves. The transition temperatures between the magnetically ordered states (AFM, AFM+FM, FM) were taken as the inception of the strong rise observed on warming. The Néel temperature was determined as the peak position of the thermomagnetic $M(T)$ data. The vertical dashed line delimits the onset of pure FM phase. The application of magnetic field yields an almost linear increase of the Curie temperature $T_{\mathrm{C}}$ while the transition temperature of the $\mathrm{AFM} \rightarrow \mathrm{AFM}+\mathrm{FM}$ $(\mathrm{AFM}+\mathrm{FM} \rightarrow \mathrm{FM})$ transformation shows the opposite trend upon increasing external field. The Néel temperature $T_{\mathrm{N}}$ is weakly sensitive to the magnetic field and slightly shifts to lower temperatures with increasing applied magnetic field. Besides the three well distinct magnetic regions - AFM, FM, and PM - a striking characteristic of the present magnetic phase diagram is the low-temperature regime where the pseudo-ternary compound $\mathrm{La} 0.9 \mathrm{Ce}_{0.1} \mathrm{Fe}_{12} \mathrm{~B}_{6}$ may be in an AFM, FM, or magnetically heterogeneous AFM+FM state depending on the magnitude of the applied magnetic field and thermomagnetic history effects. For instance, when La $0.9 \mathrm{Ce}_{0.1} \mathrm{Fe}_{12} \mathrm{~B}_{6}$ is cooled from the paramagnetic region down to $2 \mathrm{~K}$ in the absence of magnetic field and subsequently magnetized by the application of a $3 \mathrm{~T}$ field, the intermetallic compound will stay in the pure AFM ground state at $2 \mathrm{~K}$ and $3 \mathrm{~T}$. But, when cooled from high temperatures down to $2 \mathrm{~K}$ in an applied magnetic field of $3 \mathrm{~T}$, the system will go across the AFM-FM phase boundary and endure a partial $\mathrm{AFM} \rightarrow \mathrm{FM}$ magnetic phase transformation; in such experimental conditions the alloy will stay in the mixed phase (or phase separated) AFM+FM state. Another salient feature of the phase diagram is the presence of critical point around $32 \mathrm{~K}$ and $1.5 \mathrm{~T}$ at the crossover of the PM, FM, and AFM phase.

Figure 3 presents the magnetization isotherms, $M(H)$, of $\mathrm{La}_{0.9} \mathrm{Ce}_{0.1} \mathrm{Fe}_{12} \mathrm{~B}_{6}$ at some selected temperatures. For each isothermal magnetization curve, the external applied field was cycled between 0 and the maximum attainable value of $10.5 \mathrm{~T}$. Each $M(H)$ isotherm begins from the virgin state after zero-magnetic-field cooling from room temperature. Fig. 3a shows the data between 2 and $25 \mathrm{~K}$, and Fig. $3 \mathrm{~b}$ illustrates the isotherms taken at temperatures ranging between 30 and $50 \mathrm{~K}$. The virgin curves or first magnetization curves exhibits a metamagnetic phase transformation between the PM and FM states above $T_{\mathrm{N}}$ and from the AFM state into the FM state below $T_{\mathrm{N}}$. This field-induced metamagnetic transition proceeds through a gradual transformation of the PM and AFM phases into FM domains with increasing applied magnetic field. The magnetic-field-driven AFM-FM and PM-FM phase transformations are accompanied 
by large magnetic hysteresis, indicating the first-order character of the transition. The width of the hysteresis is estimated to be about $4.1 \mathrm{~T}$ at $8 \mathrm{~K}$ and progressively decreases upon heating.

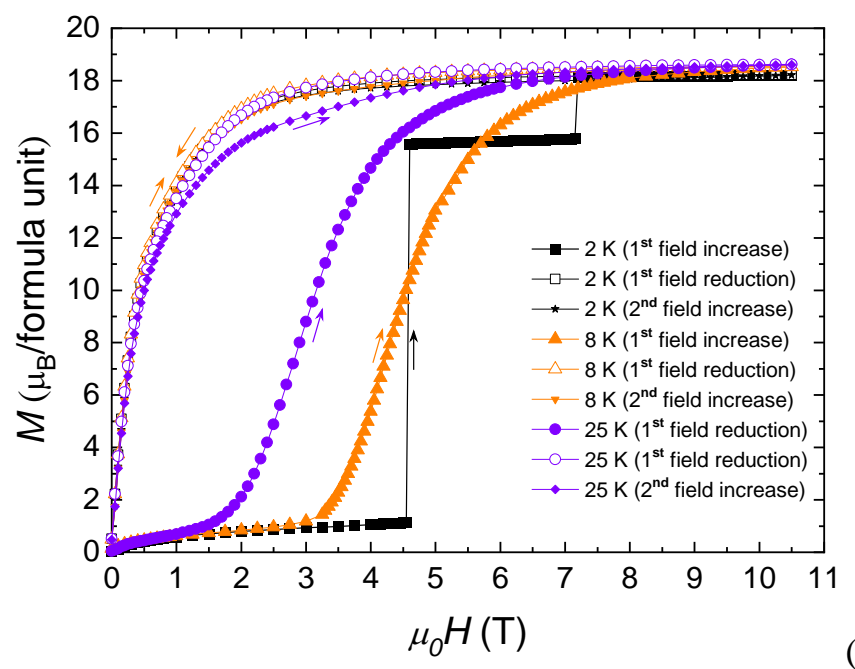

(a)

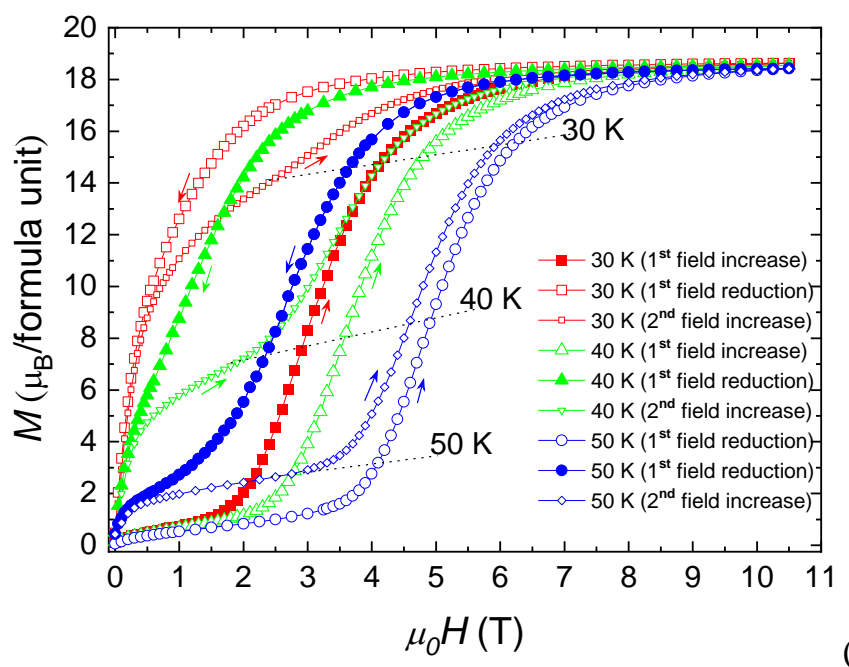

(b)

FIG. 3. Magnetization isotherms of $\mathrm{La}_{0.9} \mathrm{Ce}_{0.1} \mathrm{Fe}_{12} \mathrm{~B}_{6}$ at (a) 2,8 and $25 \mathrm{~K}$, and (b) 30,40 and $50 \mathrm{~K}$.

During the first application of the magnetic field, at $2 \mathrm{~K}$ the magnetization displays two ultrasharp steps followed by plateaus; generating an avalanche-like metamagnetic process. These staircase-like transitions arises from conversion of fraction of the sample from the AFM state into the FM state. The mixed phase AFM+FM or magnetically heterogenous state (phase separated into AFM and FM domains) is associated with the first magnetization plateau. The saturation magnetization of the fully FM polarized state is found to be $18.22 \mu_{\mathrm{B}} /$ formula unit. Neither abrupt jumps nor smooth transitions are observed in the subsequent envelope upon decreasing magnetic field. The sharpness of the multistep behavior is reduced with increasing temperature and vanishing at $8 \mathrm{~K}$ where the magnetization process becomes smooth.

During the second magnetic field increase at temperatures ranging from 2 to $25 \mathrm{~K}$, the 
curve practically coincides with the demagnetization path showing a conventional ferromagnetic magnetization process. After the applied field is reduced to zero, nearly $100 \%$ of the $\mathrm{La}_{0.9} \mathrm{Ce}_{0.1} \mathrm{Fe}_{12} \mathrm{~B}_{6}$ intermetallic remains in the forced FM phase demonstrating that the firstorder AFM-FM transformation is completely irreversible in this temperature interval. However, between 30 and $50 \mathrm{~K}$, the shape of the isothermal magnetization curves during the second increase of the applied field is even more complex and atypical when compared to the process observed below $25 \mathrm{~K}$. One can clearly observe a ferromagnetic-like dependence in the lowmagnetic-field portion (see the dashed lines in Fig. 3b) followed by a metamagnetic phase transformation. From 30 to $50 \mathrm{~K}$, the field-driven AFM-FM and PM-FM transformations are partially reversible; a percentage of the sample recovers the original AFM or PM state when the magnetic field is removed. Both reversible and irreversible transitions exist in the temperature region between 30 and $50 \mathrm{~K}$, and the proportion of the system, which endures the irreversible transformation, decreases upon heating. Above $55 \mathrm{~K}$, the PM-FM metamagnetic transformation becomes fully reversible, still with a magnetic hysteresis.

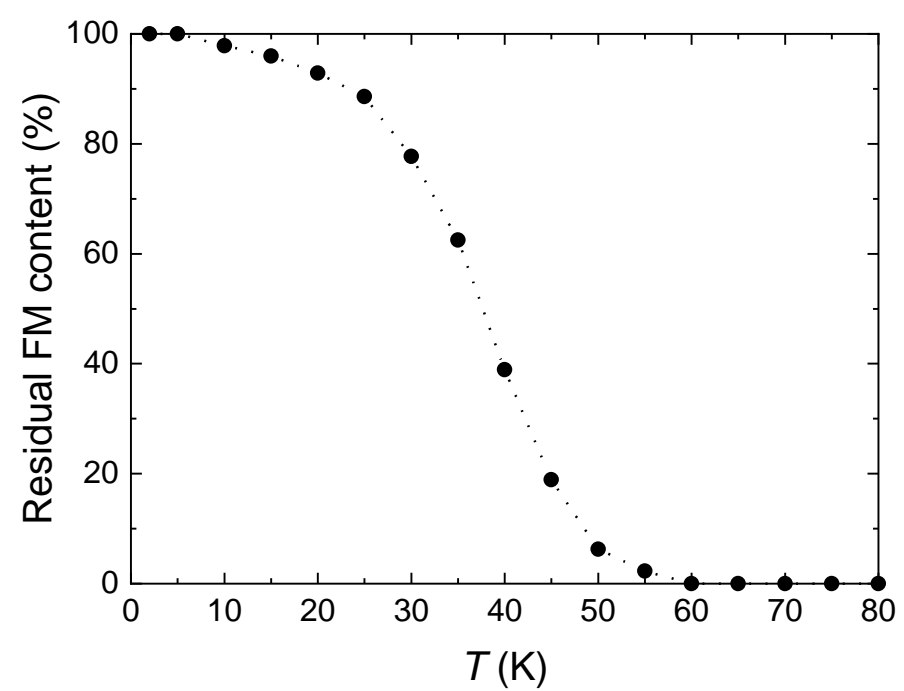

FIG. 4. Thermal variation of the amount of the residual $\mathrm{FM}$ phase in the $\mathrm{La}_{0.9} \mathrm{Ce}_{0.1} \mathrm{Fe}_{12} \mathrm{~B}_{6}$ sample after initial magnetization at $2 \mathrm{~K}$. The dashed line is the guide for the eye.

To gain a deeper insight into the relationship between the three different magnetic phases, we have studied the thermal evolution of the fraction of the AFM (PM) phase recovered from the forced FM state. For such measurements, a different procedure was used : the sample was thermally demagnetized at room temperature and zero field cooled down to $2 \mathrm{~K}$. A $10.5 \mathrm{~T}$ magnetic field was applied to magnetized the system, subsequently it was heated up to desired measurement temperature in zero magnetic field and isothermal magnetization data were collected. The concentration of the residual FM phase was assessed from the extrapolated 
magnetization value (see dashed lines in Fig. 3b) which represent the saturation magnetization in low fields without the metamagnetic-like transition. This method is similar to that used by Levin et al. ${ }^{18}$ The obtained results are shown in Fig. 4 as a function of temperature. In the temperature range between 2 and $25 \mathrm{~K}$, the change in the residual FM phase content is small and then it decreases rapidly with increasing temperature approaching zero around $55 \mathrm{~K}$.
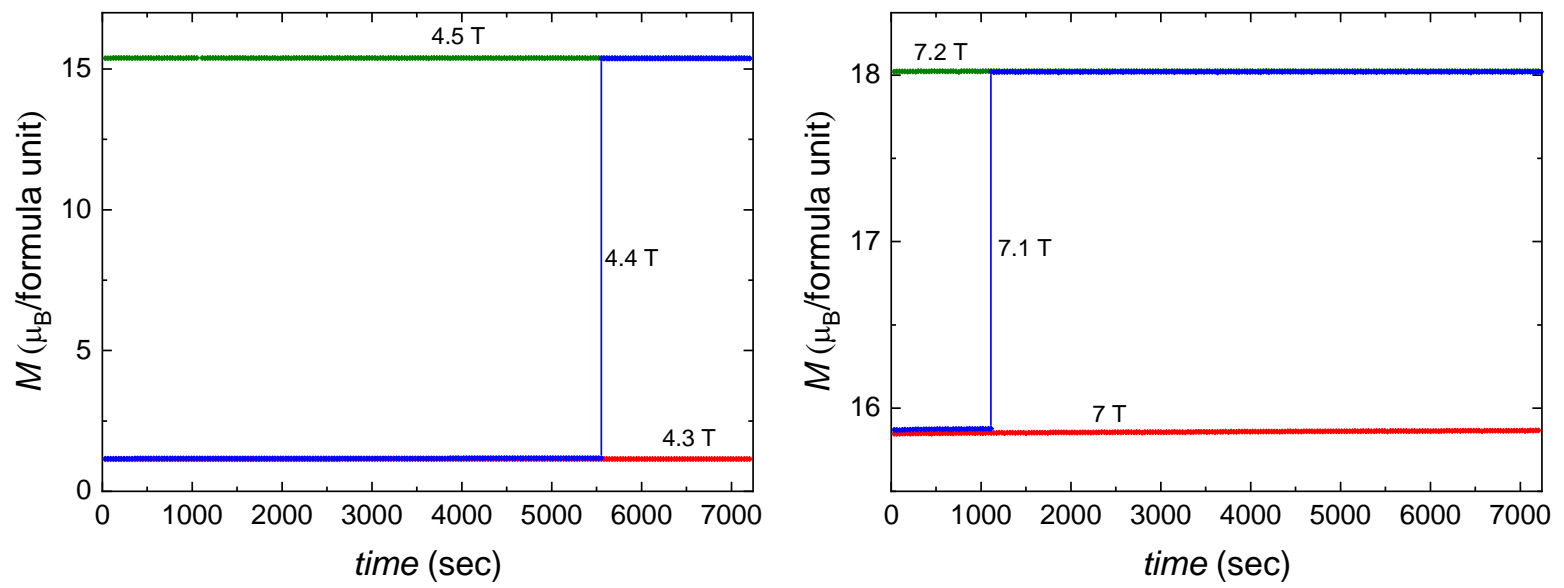

FIG. 5. Magnetization as a function of time at $2 \mathrm{~K}$ in different applied magnetic fields for $\mathrm{La}_{0.9} \mathrm{Ce}_{0.1} \mathrm{Fe}_{12} \mathrm{~B}_{6}$.

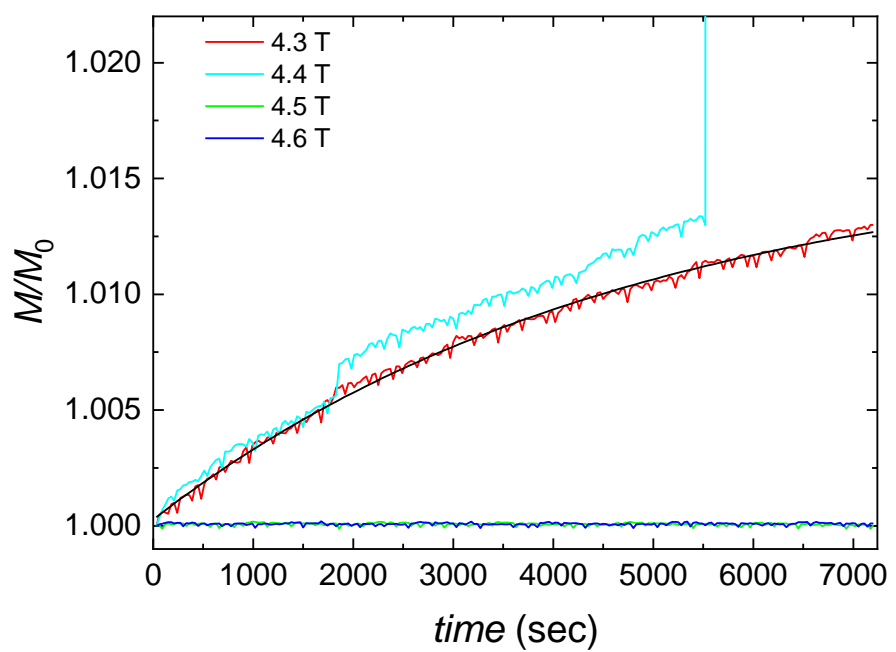

FIG. 6. Relative variation of the magnetization of $\mathrm{La}_{0.9} \mathrm{Ce}_{0.1} \mathrm{Fe}_{12} \mathrm{~B}_{6}$ as a function of time ( $M_{0}$ is the initial magnetization measured at $t=0$ ), in magnetic fields ranging from 4.3 to $4.6 \mathrm{~T}$ at $2 \mathrm{~K}$. The black solid line is fitting the curve recorded for the field value of $4.3 \mathrm{~T}$.

Considering the metastable character of the different magnetic states and the anomalous features observed in the magnetization isotherms at very low temperatures, we further investigated the time evolution of the magnetization in order to probe relaxation phenomenon and the dynamics of the magnetization steps. The magnetic relaxation measurements were performed at $2 \mathrm{~K}$ and in magnetic fields slightly below and above the critical fields of the steplike metamagnetic transitions. The time dependence of the magnetization is reported in Fig. 5. 
In an applied field of $4.3 \mathrm{~T}$ and $7 \mathrm{~T}$, the magnetization is about $1.15 \mu \mathrm{B} /$ formula unit and 15.85 $\mu_{\mathrm{B}} /$ formula unit. respectively all along the magnetization measurement process (through the holding time of the field). By contrast, when the magnetic field is increased to $4.4 \mathrm{~T}(7.1 \mathrm{~T})$ the magnetization instantaneously varies from 1.18 to $15.40 \mu_{\mathrm{B}}$ /formula unit (15.88 to 18.02 $\mu_{\mathrm{B}} /$ formula unit.) after an incubation time or transition time of $5550 \mathrm{~s}$ (1080 s). The sudden magnetization jumps observed during isothermal holding take place within a very short period of time, i.e., < $30 \mathrm{~s}$. When the relaxation measurement is repeated in magnetic fields above the critical fields, the magnetization magnitude equates the post-jump value at $4.4 \mathrm{~T}(7 \mathrm{~T})$ and is essentially constant over the entire

time span of $7200 \mathrm{~s}$. Consequently, it is obvious that the transitions detected at 4.4 and $7 \mathrm{~T}$ are fully irreversible.

The normalized magnetization $M / M_{0}$ is displayed in Fig. 6 as a function of time. $M_{0}$ represents the initial magnetization value recorded at $t=0$ for each field step. The magnetic relaxation curves in applied fields $\mu_{0} H>4.4 \mathrm{~T}$, are nearly flat without perceptible time dependence. But, isothermal holding in $\mu_{0} H<4.4 \mathrm{~T}$ leads to weak time dependence and the relaxation curves can be fitted by the following expression ${ }^{19,20}$ :

$$
M\left(\mu_{0} H, t\right)=M_{0}\left(\mu_{0} H\right)+\left[M_{\infty}\left(\mu_{0} H\right)-M_{0}\left(\mu_{0} H\right)\right]\left[1-\exp \left(-\frac{t}{\tau\left(\mu_{0} H\right)}\right)\right]
$$

where $M_{\infty}$ is the magnetization at $t=\infty$ and $\tau$ corresponds to the relaxation time directly connected to the energy barrier separating two metastable states ${ }^{21-24}$. The obtained relaxation time $\tau$ amounts to $4720 \mathrm{~s}$. It is worth to notice that the $\tau$ values in some manganese oxides showing similar characteristics vary from 2500 to $7100 \mathrm{~s}^{19,21}$ that is the same time range.

\section{B. Transport properties}

In itinerant-electron metamagnets, transport behaviors are strongly related to magnetism. The intermetallic compound $\mathrm{La}_{0} \mathrm{Ce}_{0.1} \mathrm{Fe}_{12} \mathrm{~B}_{6}$ displays multiple magnetic transformations and a remarkable metamagnetic transition which is featured by a tremendous magnetization change and a huge hysteresis. Therefore, $\mathrm{La} 0.9 \mathrm{Ce}_{0.1} \mathrm{Fe}_{12} \mathrm{~B}_{6}$ system is expected to exhibit strong magnetoresistive effects across the magnetic phase transitions. To examine more profoundly the nature of this extraordinary magnetic process (first-order character of the AFMFM and PM-FM transitions), the temperature and magnetic field dependencies of the resistivity were studied following the same experimental protocol as in the magnetization experiments described above.

Figure 7 illustrates the thermal evolution of the electrical resistivity, $\rho(T)$, of the ZFC sample under various applied fields between 0 and $8 \mathrm{~T}$. As can be seen from Fig.7, all of the 
isofield $\rho(T)$ curves present a strongly linear evolution in electrical resistivity on the hightemperature side which is reflecting the metallic character. This linear evolution is similar to that reported at high temperature for the $R \mathrm{Co}_{12} \mathrm{~B}_{6}$ compounds ${ }^{16}$ and may be attributed to the dominant electron-phonon contribution to the resistivity. In zero magnetic field, no detectable anomaly is found at $T_{\mathrm{N}}$, associated with the second-order AFM-PM transition. In a $8 \mathrm{~T}$ applied field, the large variation in resistivity around $80 \mathrm{~K}$ results from magnetic transition between the FM (low resistivity) and PM (high resistivity) states. This magnetic ordering temperature value is in accord with the Curie temperature $T_{\mathrm{C}}$ obtained from thermomagnetic data. The $\rho(T)$ curves $\left(\mu_{0} H=5\right.$ and $\left.6 \mathrm{~T}\right)$ show the same behavior as that at $8 \mathrm{~T}$ except for a negative shift of the Curie point toward lower temperatures. Around the Curie temperature, the rise of applied magnetic field leads to a lowering of the electrical resistivity owing to the decreased spin scattering by the magnetic field driven orientation of the local magnetic moments. The large change in resistivity in the vicinity of $T_{\mathrm{C}}$ is indicative of a strong interaction of Fe magnetic moments with conduction electrons.

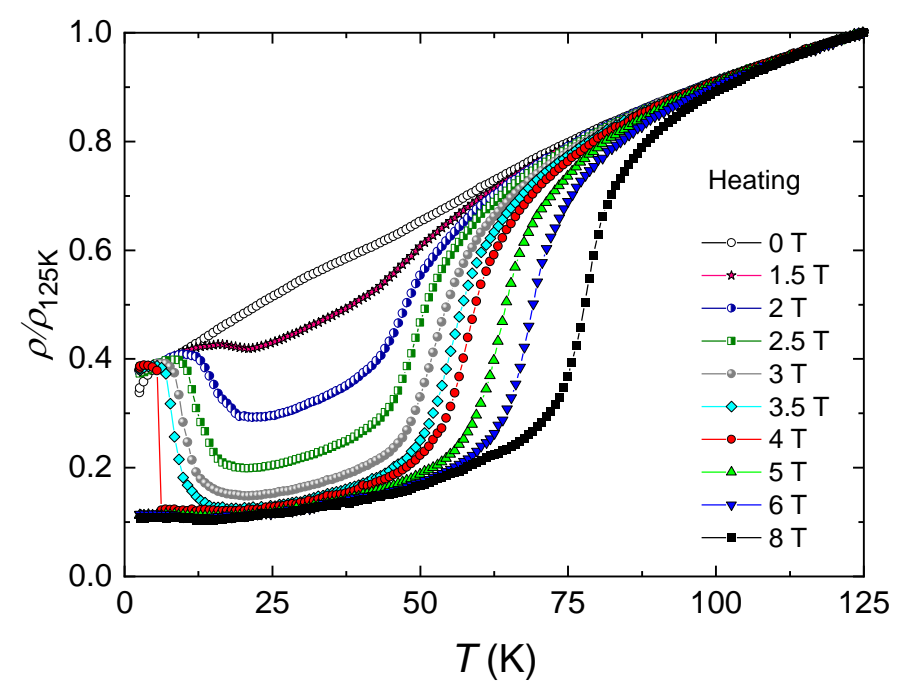

FIG. 7. Temperature dependence of the relative electrical resistivity of $\mathrm{La}_{0.9} \mathrm{Ce}_{0.1} \mathrm{Fe}_{12} \mathrm{~B}_{6}$ measure on heating in various magnetic fields.

The thermal variation of the resistivity in the magnetic field range between 1.5 and 3.5 $\mathrm{T}$ differs absolutely from the behavior seen in zero and high fields. Upon increasing temperature from $2.5 \mathrm{~K}$, the electrical resistivity first diminishes at the onset of the ferromagnetic ordering and then increases at high temperatures, giving rise to a basin-like behavior. This singular temperature dependence of the resistivity is consistent with the existence of both lowtemperature AFM-FM and high-temperature FM-PM magnetic phase transitions. Interestingly, a sharp drop is detected in the isofield $\rho(T)$ curve recorded in $4 \mathrm{~T}$; the normalized electrical 
resistivity changes abruptly from 0.38 to 0.12 . This discontinuity in the $\rho(T)$ plot is strikingly similar to that observed in the $4 \mathrm{~T}$ ZFC $M(T)$ curve and proves the strong correlation between transport and magnetic properties in $\mathrm{La} 0 .{ }_{9} \mathrm{Ce}_{0.1} \mathrm{Fe}_{12} \mathrm{~B}_{6}$. The significant resistivity variation across the order-order $\mathrm{AFM} \rightarrow \mathrm{FM}$ magnetic transformation can be ascribed to the difference in the strength of the scattering of the conduction electrons by the AFM and FM magnons and by the phonons. Our experimental results denote that the scattering in the AFM spin configuration is much larger than that in the FM structure.

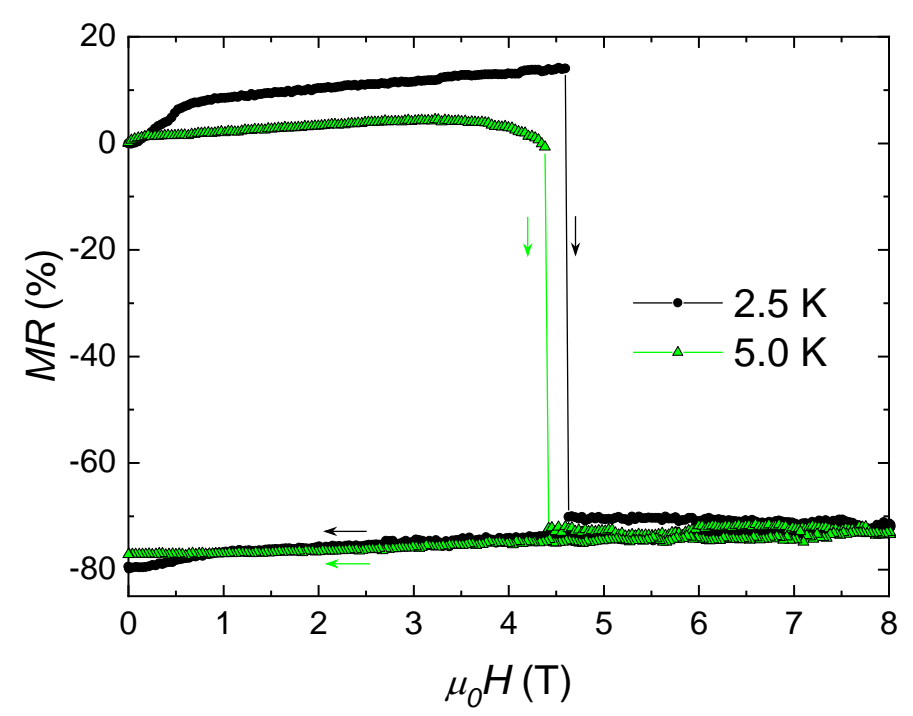

FIG. 8. Magnetoresistance isotherm of $\mathrm{La}_{0.9} \mathrm{Ce}_{0.1} \mathrm{Fe}_{12} \mathrm{~B}_{6}$ at 2.5 and $5 \mathrm{~K}$.

In order to get a better understanding of the interplay between charge and magnetic degrees of freedom in this compound, magnetic-field-dependent resistivity measurements were performed at various fixed temperatures. From these data, the magnetoresistance ratio MR was evaluated as $\left[\rho\left(\mu_{0} H, T\right)-\rho(0, T)\right] / \rho(0, T)$. The corresponding results are reported in Figs. 8-9 for different representative temperature intervals. At very low temperatures - Fig. 8 - the fieldincreasing branch of the isothermal magnetoresistance curve exhibits ultrasharp stepwise change in the same magnetic field range where a sharp discontinuity was found in the magnetization data. This steep and substantial variation in the electrical resistivity is due to the first-order AFM-FM transition. The resistivity is larger in the AFM ground state when compared with that in the forced FM phase. The reverse leg presents no transition since the compound remains in the field-induced FM state as mentioned above, thus confirming the interpretation of the magnetization curves. After bringing the applied magnetic field back to zero, a remanent (nonzero) magnetoresistance is present simply meaning that the initial resistivity value is not recovered. This experimental observation endorses the conclusion that 
the metamagnetic transition is totally irreversible in the very low temperature regime. To restore the virgin state, and thus, the initial resistivity value, it is necessary to warm up the system well beyond $T_{\mathrm{C}}$ and subsequently cooled it down in zero field.

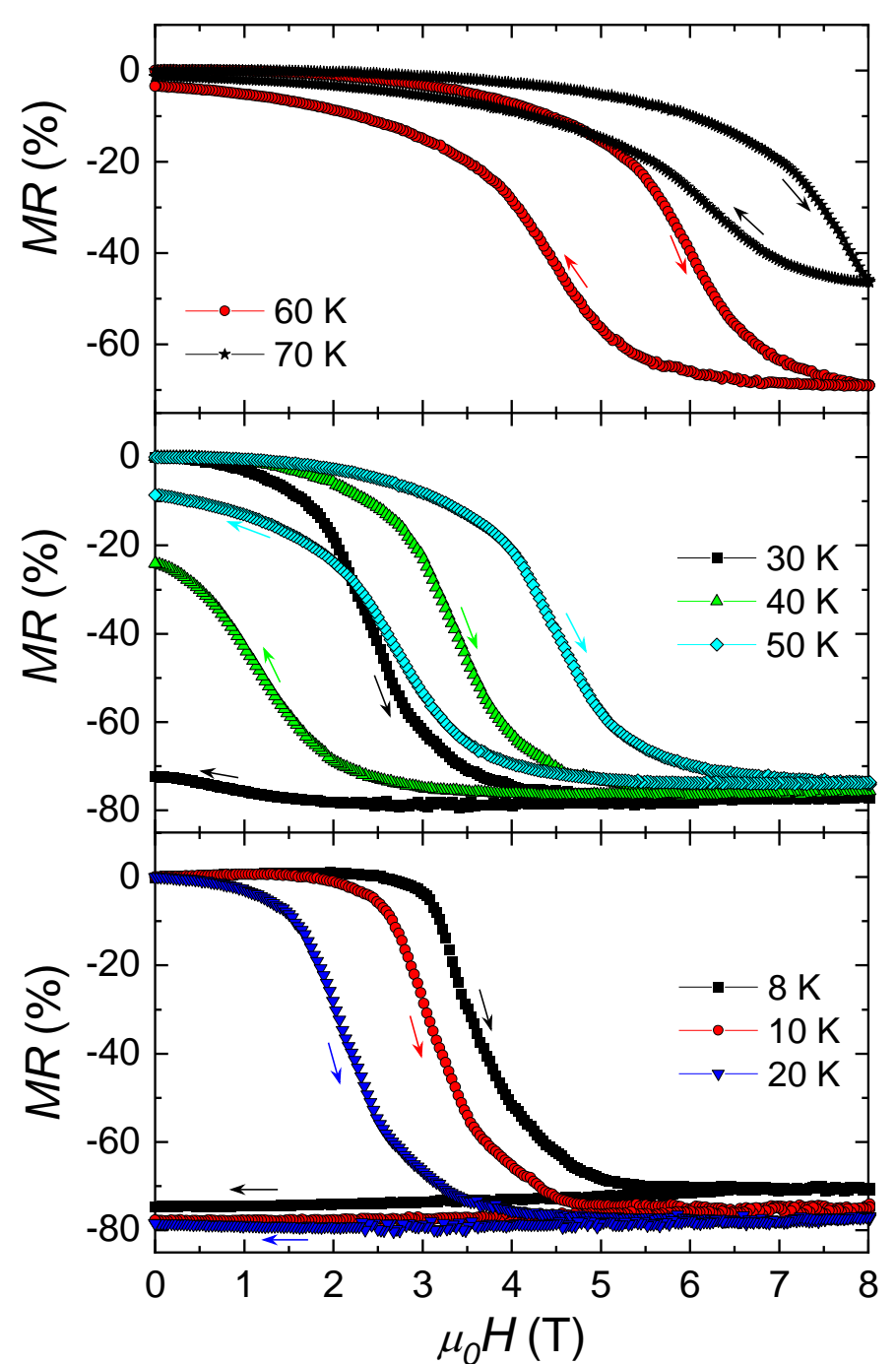

FIG. 9. Magnetoresistance isotherms of $\mathrm{La}_{0.9} \mathrm{Ce}_{0.1} \mathrm{Fe}_{12} \mathrm{~B}_{6}$ for the temperature intervals 8 to $20 \mathrm{~K}$ (bottom panel), 30 to $50 \mathrm{~K}$ (middle panel) and 60 to $70 \mathrm{~K}$ (top panel).

In the temperature range from 8 to $70 \mathrm{~K}$ (Fig. 9), the magnetoresistance shows progressive variations across both AFM-FM and PM-FM transitions differently from the discontinuous process observed at 2.5 and $5 \mathrm{~K}$. The magnetoresistance isotherms display huge magnetic hysteresis which is one of the signatures of a first-order transition. This hysteretic character and the irreversibility/reversibility depend on the temperature interval. Three distinct representative temperature intervals are represented in Fig. 9: from 8 to $20 \mathrm{~K}$ (bottom panel), between 30 and $50 \mathrm{~K}$ (middle panel) and $T \geq 60 \mathrm{~K}$ (top panel). In the AFM ground state, for example at $10 \mathrm{~K}, \mathrm{MR}$ is very small at low fields but drastically declines above the transition 
field as the compound undergoes a transformation to the FM phase yielding a giant negative MR effect of about $-78 \%$. During the decreasing-field process, the electrical resistivity keeps almost the same value down to the zero magnetic field point; confirming the irreversible nature of the magnetic phase transition below $25 \mathrm{~K}$. From $\sim 30$ to $\sim 50 \mathrm{~K}$, the curve of the downwardfield run diverts from the nearly pure FM behavior and a metamagnetic-like transition occurs at lower field, leading to an evident hysteresis between both magnetic field scans. The magnetic transformation is partially reversible in this temperature interval. Above $55 \mathrm{~K}$, as exemplified by the isothermal magnetoresistance curves at 60 and $70 \mathrm{~K}$ - Fig. 9 - the initial value of the electrical resistivity is recovered after removal of the external field. As a result, the PM-FM transition becomes reversible.

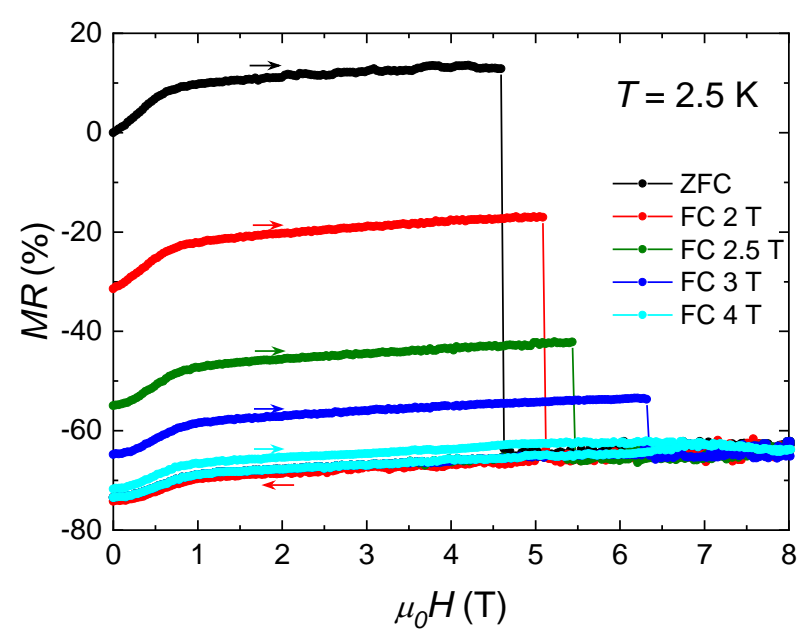

FIG. 10. Magnetoresistance isotherm of $\mathrm{La}_{0.9} \mathrm{Ce}_{0.1} \mathrm{Fe}_{12} \mathrm{~B}_{6}$ at $2.5 \mathrm{~K}$ recorded after cooling the sample in different magnetic fields.

As demonstrated by magnetic measurements, field cooling modifies considerably the relative proportion of the different magnetic phases (see Fig. 4). By analogy to the magnetization data, we have explored the influence of the magnetic field strength applied during the cooling process on the resistivity. For such studies, the intermetallic compound was cooled in magnetic field $\left(\mu_{0} H>0\right)$ from the paramagnetic region down to $2.5 \mathrm{~K}$. After the temperature of $2.5 \mathrm{~K}$ is stable, the cooling field was set to zero, and subsequently the electrical resistivity was measured against external field up to $8 \mathrm{~T}$ and back to zero magnetic field (Fig 10). This field cooling procedure diminishes remarkably the low-field electrical resistivity due to the growth of the FM phase content to the detriment of the AFM component. The critical magnetic field at which the abrupt jump takes place is completely mastered by the residual FM amount. Field cooling moves the step transition toward higher magnetic fields. Cooling in an adequately high external field transforms the sample into a fully FM polarized state, eliminating 
the resistivity jump.

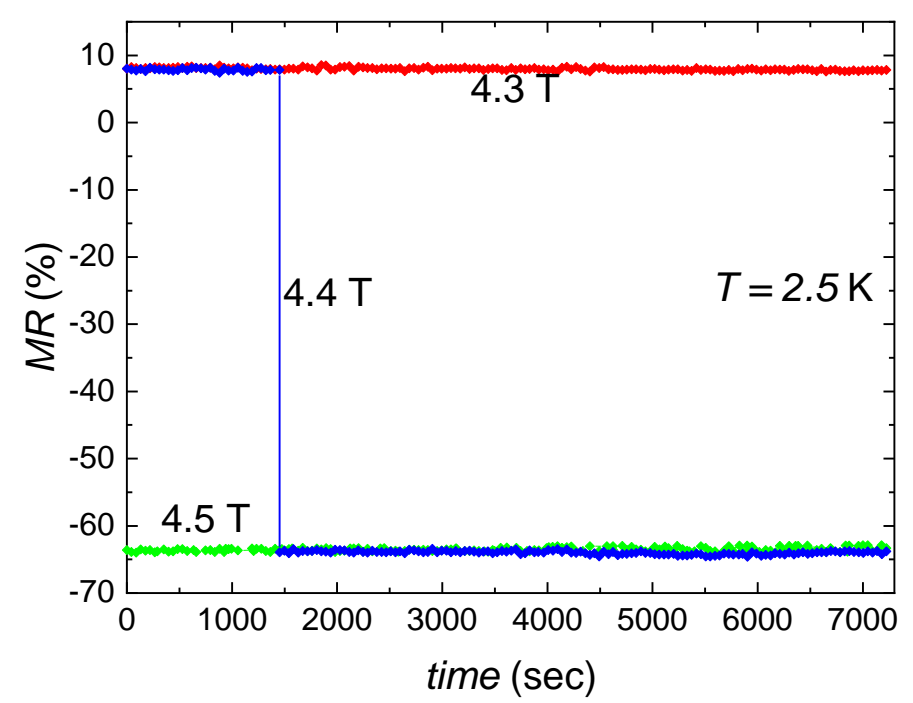

FIG. 11. Time dependence of the magnetoresistance collected at the indicated applied fields for $\mathrm{La}_{0.9} \mathrm{Ce}_{0.1} \mathrm{Fe}_{12} \mathrm{~B}_{6}$ at $2.5 \mathrm{~K}$.

Time-dependent phenomena (relaxation effects) were investigated to probe more directly the dynamics of the resistivity sharp step. The resistive relaxation experiments were carried out at $2.5 \mathrm{~K}$ and in magnetic fields around the critical field of the sharp step seen in the isothermal magnetoresistance curve. Prior to these time dependence measurements, the system was zero field cooled from ambient temperature down to $2.5 \mathrm{~K}$. After the temperature stabilization, a magnetic field is applied and then the resistivity is measured versus time (total duration of $7200 \mathrm{~s}$ ). The same experimental procedure was repeated several times applying various fields in steps of $0.1 \mathrm{~T}$, and the obtained results are represented in Fig. 11. For $\mu_{0} H=$ 4.4 T, a giant resistive relaxation effect is detected, the magnetoresistance drops spontaneously from $\approx 8 \%$ to $\approx-64 \%$, due to, the burst-like development of FM domains inside the AFM matrix. It is noteworthy that this outstanding and steep resistivity jump during isothermal holding occurs within a time period smaller than the time interval separating two consecutive measurement points. The most prominent distinctive characteristic in the relaxation experiments is the colossal impulsive jump in resistivity at a given time when both external parameters (magnetic field and temperature) are kept constant. The incubation time deduced from the $4.4 \mathrm{~T}$ resistive relaxation curve is equal to $1450 \mathrm{~s}$. The quality of the temperature stabilization was inspected throughout the isothermal holding ( $2.500 \pm 0.005 \mathrm{~K})$. Furthermore, the magnetic field is equally expected to be very stable over the entire duration of the relaxation experiments since it is generated by a superconducting magnet in its persistent mode. Remarkably, this peculiar stepwise resistive relaxation phenomenon found in $\mathrm{La} 0 .{ }_{9} \mathrm{Ce}_{0 .} \mathrm{Fe}_{12} \mathrm{~B}_{6}$ shows a similitude to the singular behavior seen in conventional martensitic transitions. The 
time dependence of the electrical resistivity for the Fe-31.7at.\% Ni alloy ${ }^{25}$ is impressively similar to Fig. 10, i.e., a sudden step after an incubation time of $1020 \mathrm{~s}$.

Although the spontaneous jump is observed on both resistive and magnetic relaxation curves recorded in the same applied field of $4.4 \mathrm{~T}$, however, there is a large discrepancy in the incubation time. The transition time significantly differs from $5550 \mathrm{~s}$ to $1450 \mathrm{~s}$ for magnetic and resistive isothermal holding, respectively. This reveals that the characteristic time associated with the abrupt jump does not constitute a material constant. The phenomenal relaxation effects found at $4.4 \mathrm{~T}$ resemble an explosive instability, where the magnetization and the resistivity of the compound undergo a tremendous variation within a very short interval of time. The consistency found in the magnetic and magnetotransport properties clearly demonstrates the strong coupling between charge and spin degrees of freedom in $\mathrm{La}_{0.9} \mathrm{Ce}_{0.1} \mathrm{Fe}_{12} \mathrm{~B}_{6}$ itinerant-electron metamagnet.

\section{CONCLUSION}

We have presented the experimental results of a systematic investigation of both the magnetic and electronic transport properties, including magnetotransport ones, of La0.9 $\mathrm{Ce}_{0.1} \mathrm{Fe}_{12} \mathrm{~B}_{6}$ as a function of applied magnetic field, temperature, and time. The intermetallic compound $\mathrm{La}_{0.9} \mathrm{Ce}_{0.1} \mathrm{Fe}_{12} \mathrm{~B}_{6}$ displays many salient features in the magnetization and resistivity data including a strong magnetic-field-driven irreversibility and multiple avalanche-like metamagnetic transitions at low temperatures. The magnetic-field-induced firstorder AFM-FM and PM-FM metamagnetic transitions are accompanied by giant negative magnetoresistance effects $(\mathrm{MR}=-78 \%)$. The most extraordinary phenomena concerning $\mathrm{La} 0 .{ }_{9} \mathrm{Ce}_{0.1} \mathrm{Fe}_{12} \mathrm{~B}_{6}$ is that the evolutions of the electrical resistivity and the magnetization as a function of time undergo a spontaneous transition after a long incubation period when both external parameters, temperature and applied magnetic field, are kept constant. These peculiar stepwise magneto-resistive relaxation effects observed in $\mathrm{La}_{0 .} \mathrm{Ce}_{0.1} \mathrm{Fe}_{12} \mathrm{~B}_{6}$ shed light on the phenomenon of staircase-like transitions reported for various classes of materials. We conclude that the $\mathrm{La} 0.9 \mathrm{Ce}_{0.1} \mathrm{Fe}_{12} \mathrm{~B}_{6}$ compound exhibits unconventional magnetic properties resulting from an itinerant-electron system featured by AFM and FM states close in energy. The unusual metamagnetic process manifests itself also in the magnetoresistance behavior.

\section{DATA AVAILABILITY}

The data that support the findings of this study are available from the corresponding author upon reasonable request. 


\section{References}

${ }^{1}$ L. V. B. Diop, O. Isnard, and J. Rodríguez-Carvajal, Phys. Rev. B 93, 014440 (2016).

${ }^{2}$ S. Fujieda, K. Fukamichi, and S. Suzuki, J. Magn. Magn. Mater. 421, 403 (2017).

${ }^{3}$ L. V. B. Diop, and O. Isnard, Appl. Phys. Lett. 108, 132401 (2016).

${ }^{4}$ L. V. B. Diop, and O. Isnard, Phys. Rev. B 97, 014436 (2018).

${ }^{5}$ L. V. B. Diop, and O. Isnard, J. Appl. Phys. 119, 213904 (2016).

${ }^{6}$ L. V. B. Diop, O. Isnard, Z. Arnold, J.P. Itié, J. Kastil, and J. Kamarad, Solid State Comm. 252, 29 (2017).

${ }^{7}$ G. I. Miletic, and Z. Blazina, J. Magn. Magn. Mater. 323, 2340 (2011).

${ }^{8}$ G. I. Miletic, and Z. Blazina, J. Alloys Compd. 430, 9 (2007).

${ }^{9}$ M. Rosenberg, T. Sinnemann, M. Mittag, and K. H. J. Buschow, J. Alloys Compd. 182, 145 (1992)

${ }^{10}$ Q. A. Li, C. H. de Groot, F. R. de Boer, and K. H. J. Buschow, J. Alloys Compd. 256, 82 (1997).

${ }^{11}$ M. Mittag, M. Rosenberg, and K. H. J. Buschow, J. Magn. Magn. Mater. 82, 109 (1989).

${ }^{12}$ K. H. J. Buschow, D. B. de Mooij, and H. M. van Noort, J. Less-Common Met. 125, 135 (1986).

${ }^{13}$ K. Niihara, and S. Yajima, Chem. Lett. 1, 875 (1972).

${ }^{14}$ Yu. B.Kuz'ma, G.V. Chernyak, and N. F. Chaban, Dopov. Akad. Nauk. Ukr. RSR Ser. A 12, $80(1981)$

${ }^{15}$ W. Jung, and D. Quentmeier, Z. Kristallogr. 151, 121 (1980).

${ }^{16}$ F. Mesquita, S. G. Magalhaes, P. Pureur, L. V. B. Diop, and O. Isnard, Phys. Rev. B 101, 224414 (2020).

${ }^{17}$ A. Barlet, J. C. Genna, and P. Lethuillier, Cryogenic 31, 801 (1991).

${ }^{18}$ E. M. Levin, K. A. Gschneidner, Jr., and V. K. Pecharsky, Phys. Rev. B 65, 214427 (2002).

${ }^{19}$ V. Hardy, A. Maignan, S. Hebert, C. Yaicle, C. Martin, M. Hervieu, M. R. Lees, G. Rowlands, D. M. Paul, and B. Raveau, Phys. Rev. B 68, 220402(R) (2003).

${ }^{20}$ B. Maji, K. G. Suresh, and A. K. Nigam, Europhys. Lett. 91, 37007 (2010).

${ }^{21}$ D. Q. Liao, Y. Sun, R. Yang, Q. Li, and Z. Cheng, Phys. Rev. B 74,174434 (2006).

${ }^{22}$ O. Iglesias, and A. Labarta, Phys. Rev. B 70, 144401 (2004).

${ }^{23}$ O. Iglesias, and A. Labarta, J. Appl. Phys. 91, 4409 (2002).

${ }^{24}$ L. Balcells, O. Iglesias, and A. Labarta, Phys. Rev. B 55, 8940 (1997).

${ }^{25}$ T. Kakeshita, J. Katsuyama, T. Fukuda, and T. Saburi, Mater. Sci. Eng. A 312, 219 (2001). 


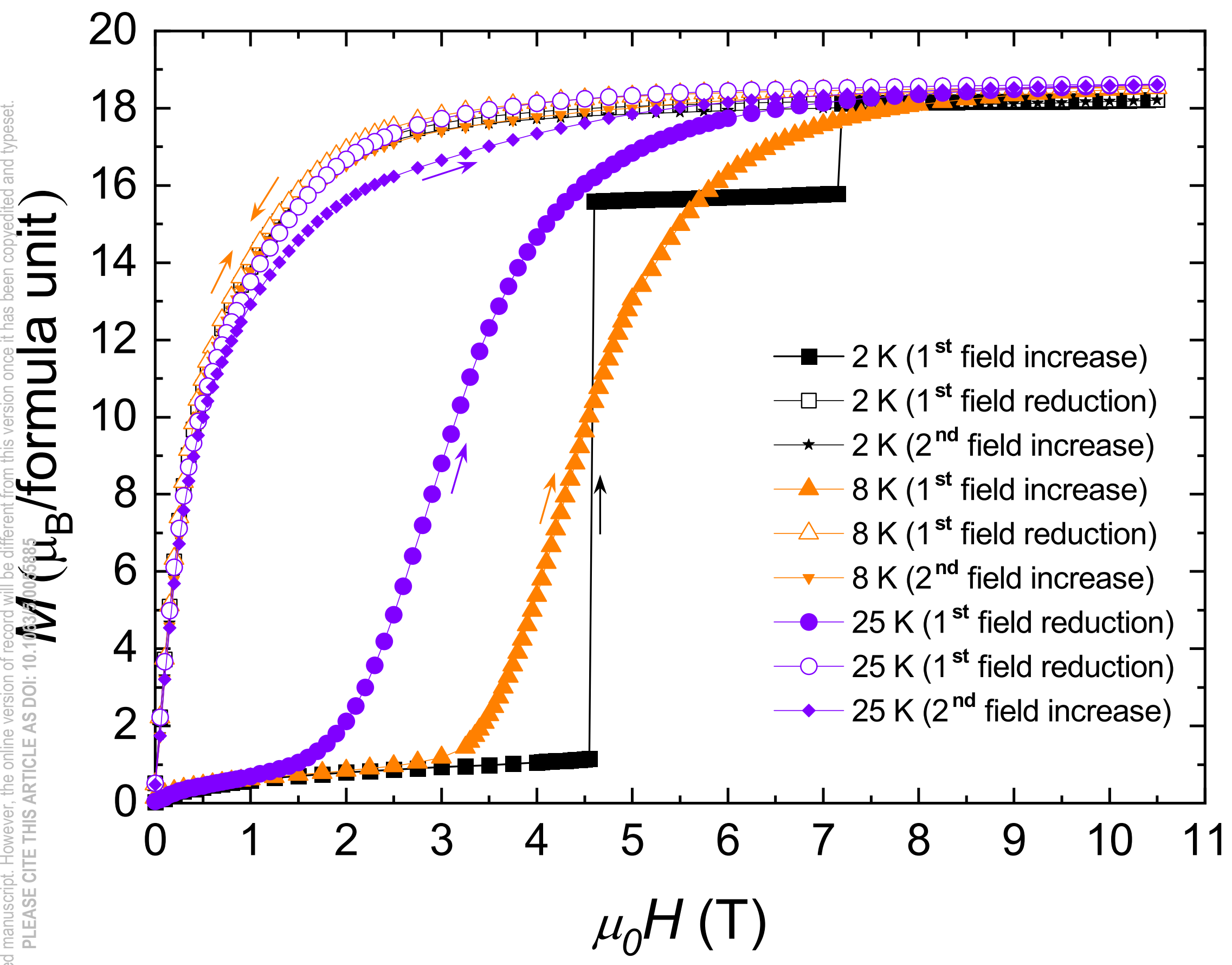




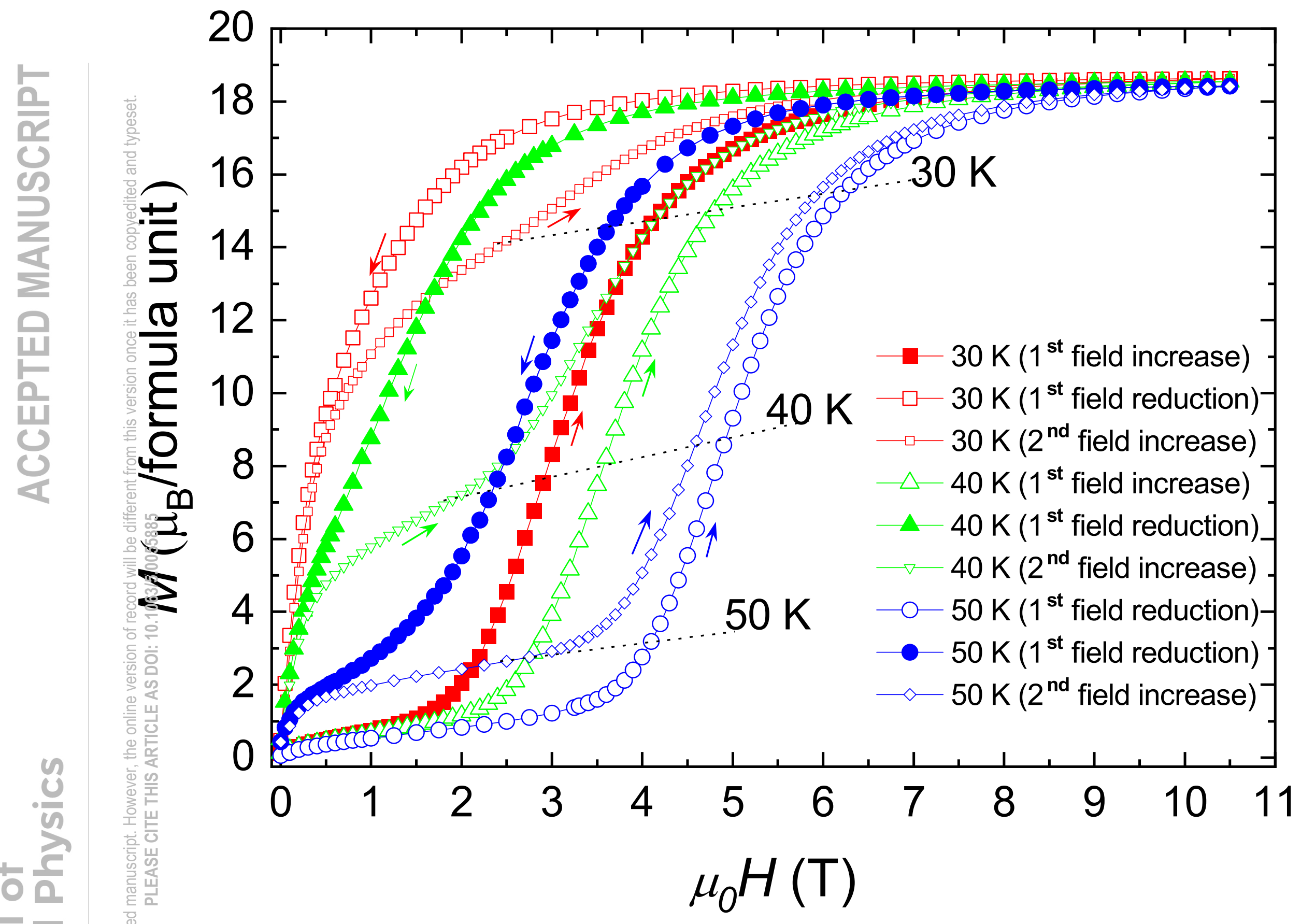




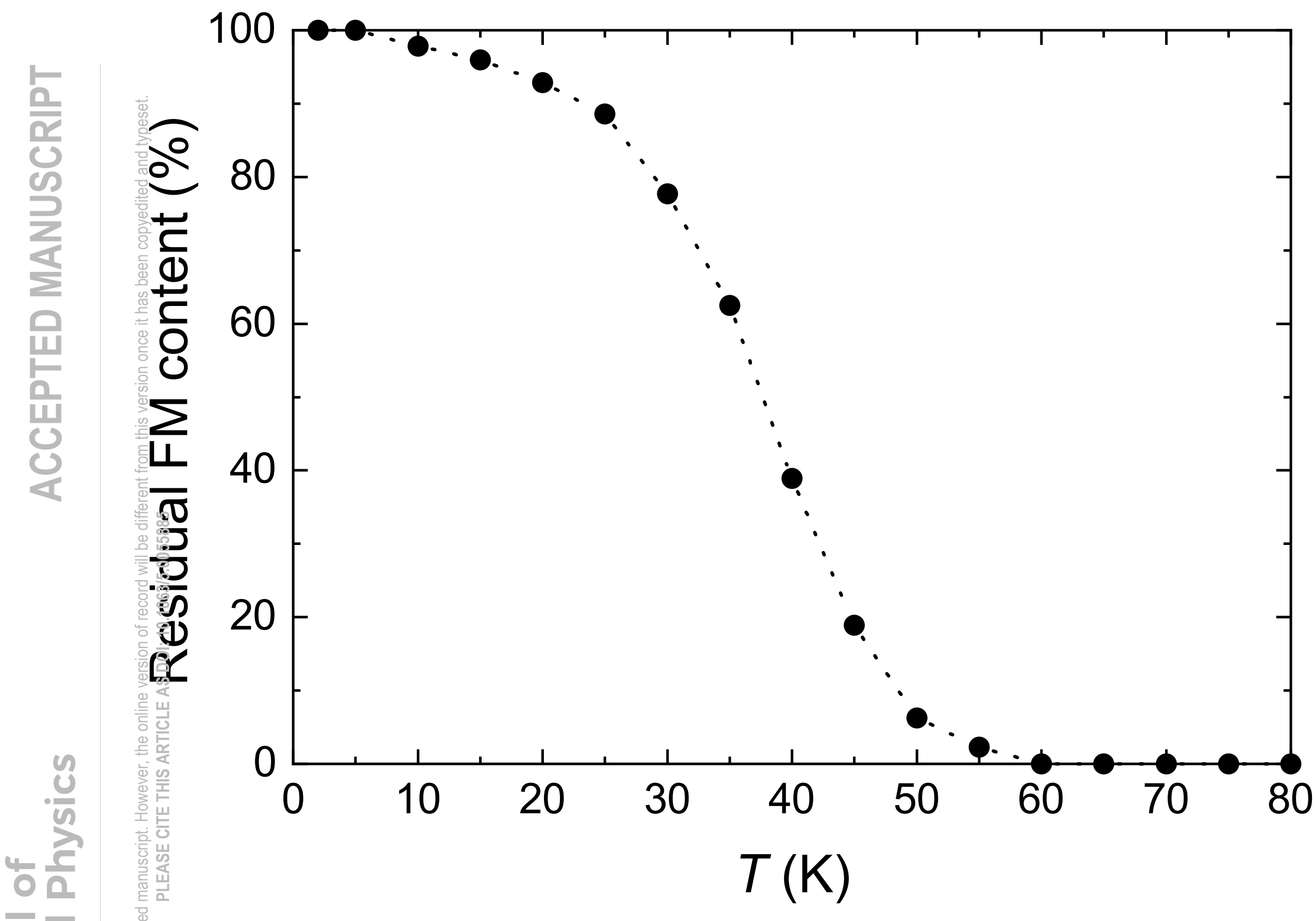




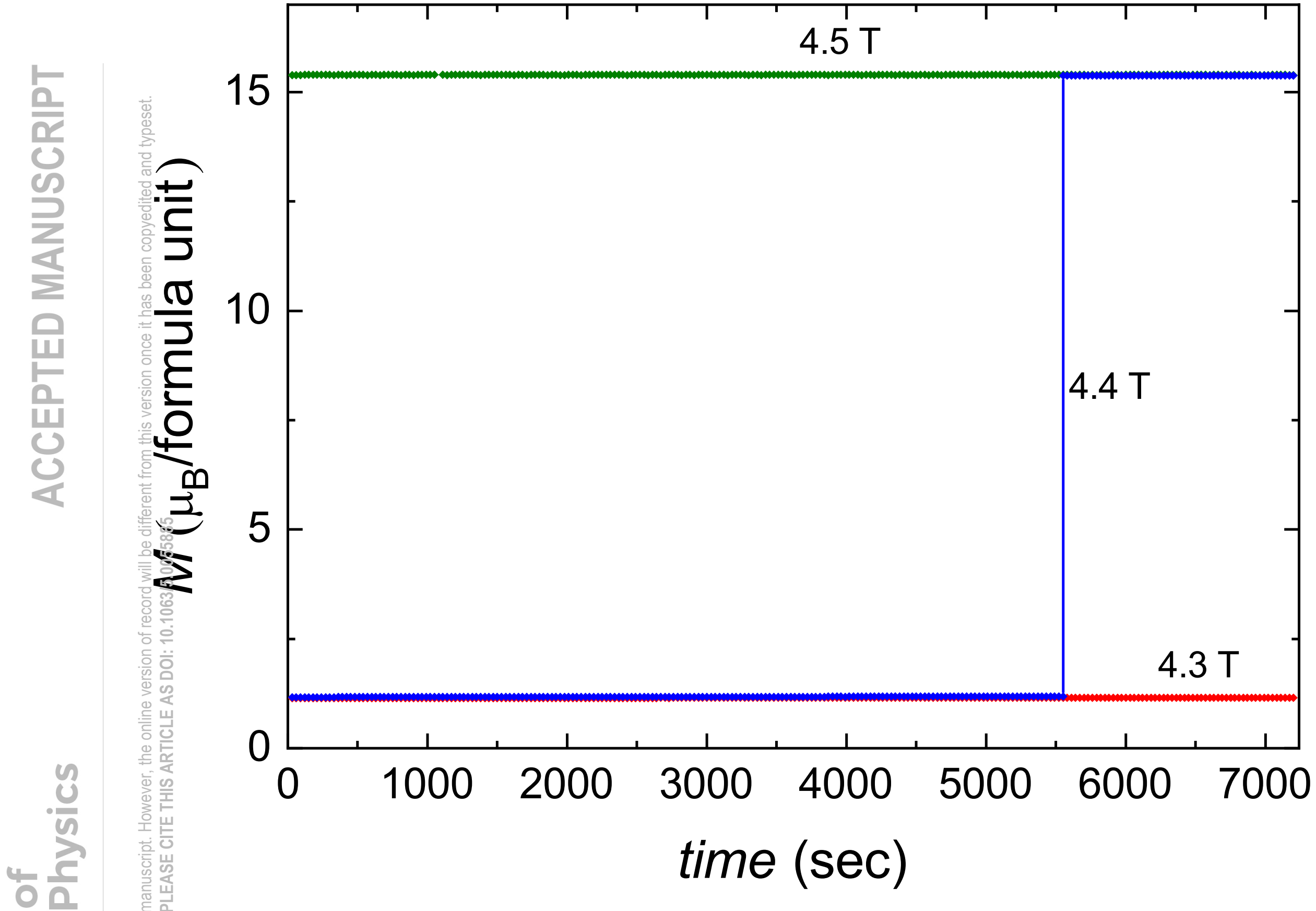




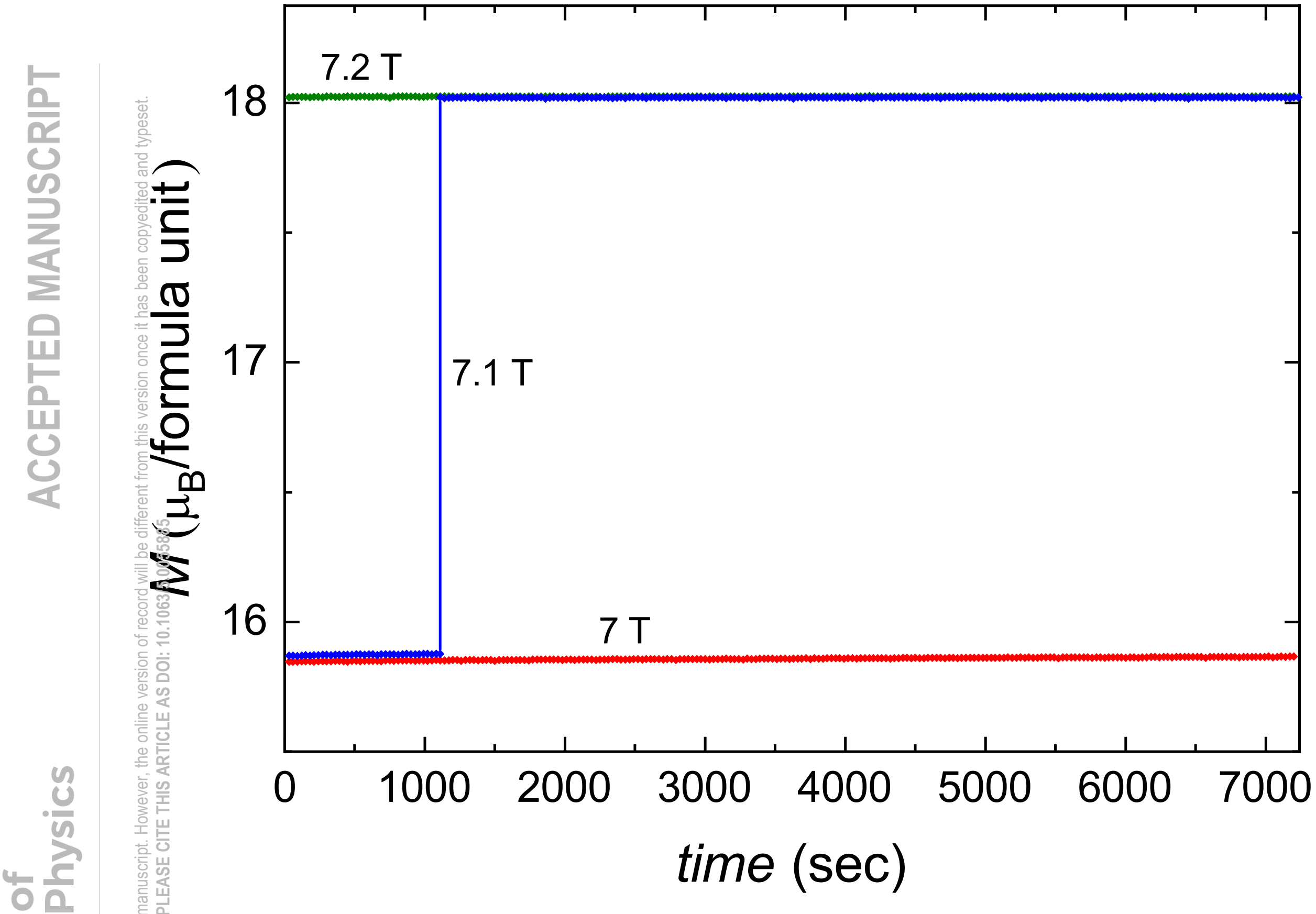




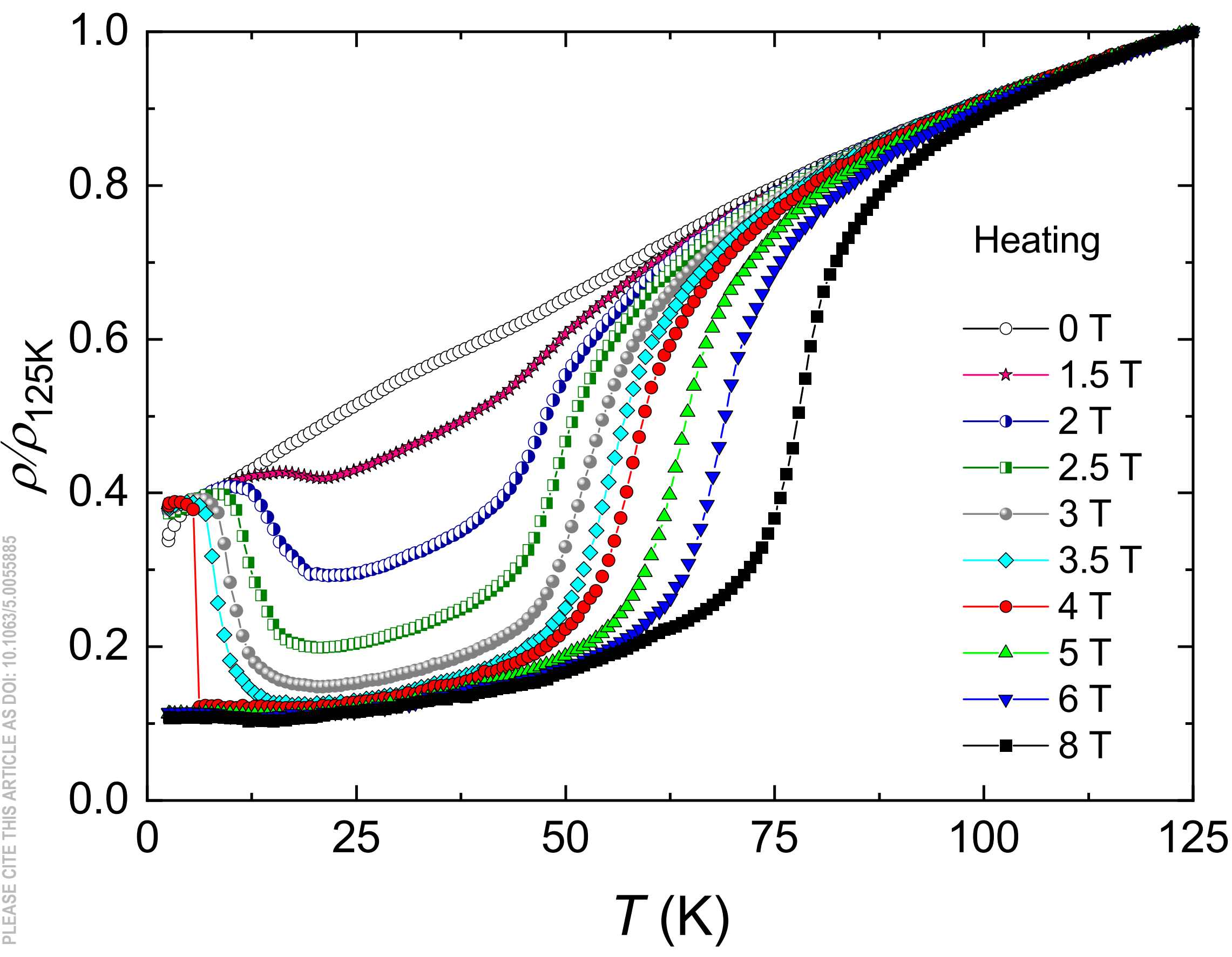




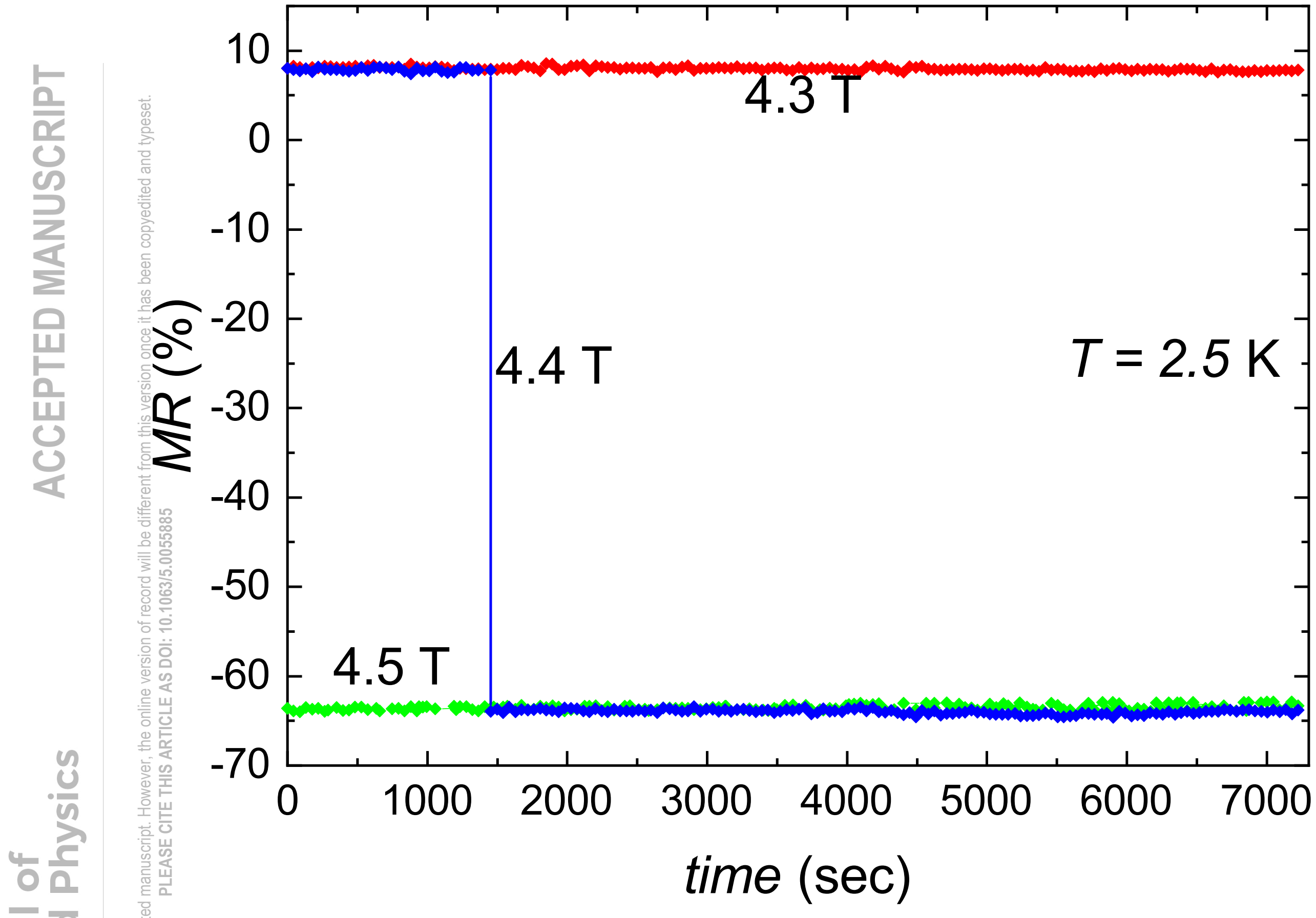

